\title{
Taxonomic affinities of Cryptocaryon irritans and Ichthyophthirius multifiliis inferred from ribosomal RNA sequence data
}

\author{
B. K. Diggles, R. D. Adlard \\ Department of Parasitology, The University of Queensland, Brisbane, Queensland 4072, Australia
}

\begin{abstract}
Comparison of partial sequences of the 18S rRNA gene of the parasitic ciliates Cryptocaryon irritans and Ichthyophthirius multifiliis confirmed that these taxa are not as closely related as was first thought. Phylogenetic trees generated from sequence data grouped I. multifilis with 3 species of Tetrahymena, supporting the existing taxonomic classification of these 2 genera together in the Order Hymenostomatida, Class Oligohymenophora. In contrast, C. irritans was grouped with Colpoda inflata (Class Colpodea) supporting the theory that the life cycle and morphological similarities evident between I. multifiliis and C. irritans are an example of convergent evolution.
\end{abstract}

KEY WORDS: Cryptocaryon tritans - Ichthyophthirius multifilis - 18S rRNA gene Molecular taxonomy

\section{INTRODUCTION}

The ciliates Cryptocaryon irritans and Ichthyophthirius multifiliis are important parasites of marine and freshwater fishes, respectively. Neither exhibit strict host specificity, but both may be highly pathogenic, cause severe epizootics and contribute to major losses of cultured and ornamental fishes. I. multifiliis, the causative agent of 'Ich', is a serious pathogen of virtually all freshwater fish in aquaria, aquaculture (Nigrelli et al. 1976) and even in the wild (Wurtsbaugh \& Tapia 1988). C. irritans causes significant problems in marine aquaria (Nigrelli \& Ruggeri 1966, Wilkie \& Gordin 1969), and in the culture of many species of marine fish (Huff \& Burns 1981, Colorni 1985, Kaige \& Miyazaki 1985, Rasheed 1989).

Cryptocaryon irritans has generally been regarded in the past as a closely related marine counterpart to Ichthyophthirius multifiliis, due mainly to the many similarities in their superficial morphologies and life cycles. In fact, C. irritans was first observed in marine aquaria by Sikama (1937), who later (Sikama 1961) described the 'new' ciliate parasite of marine fishes as Ichthyophthirius marinus due to its close resemblance to I. multifiliis, not knowing the parasite had been previously described as C. irritans by Brown (1951). Both C. irritans and I. multifiliis are parasites of the body surface and gill epithelium of fishes. Both species also exhibit a direct life cycle involving palintomic division within the cyst stage (tomont) which bursts, liberating infective stages (theronts) which mature into adult parasites (trophonts) on the host, these appearing as visible 'white-spots' on the surfaces of infected fish Because of these similarities, both species have been placed together in the family Ichthyophthiridae by some authors (Nigrelli \& Ruggeri 1966, Corliss 1975, 1979, de Puytorac 1994) whereas other authors regard C. irritans as a holotrich ciliate incertae sedis (Cannella 1972, Lom \& Dykova 1992).

Many authors (e.g. Sogin \& Elwood 1986, Lynn \& Sogin 1988, Greenwood et al. 1991) have suggested that too much emphasis has previously been placed on the classification of ciliates on the basis of morphological criteria, particularly with regard to the characterisation of ciliature and infraciliature, and that more effective methods of resolving taxonomic relationships among ciliates may be through the use of molecular techniques to acquire genomic information, such as 18S rRNA gene sequences. The present investigation was conducted to determine and compare partial sequences of the 18S rRNA gene of Ichthyophthirius multifiliis and Cryptocaryon irritans. These sequences were then compared with those of 6 other ciliate species to study their phylogenetic relationships. 


\section{MATERIALS AND METHODS}

Nucleotide sequences were determined for 3 isolates of Cryptocaryon irritans and 2 isolates of Ichthyophthirius multifiliis. Isolates of $C$. irritans tomonts were obtained from bream Acanthopagrus australis caught in the Brisbane River, Moreton Bay, Queensland, iodine bream Gymnocranius audleyi from Heron Island, Queensland, and gilt-head sea bream Sparus aurata from Eilat, Israel. Isolates of $I$. multifiliis were obtained from comet goldfish Carassius auratus from local fish distributors, and black moor goldfish $C$. auratus imported from Hong Kong and obtained during quarantine.

Tomonts of each Cryptocaryon irritans isolate were coilected with a fine paini vu ush from the bottuñ of tanks containing infected fish, fixed in $70 \%$ ethanol and stored in $100 \%$ ethanol. DNA was subsequently extracted from single tomonts using a phenol-chloroform method (Sambrook et al. 1989). Each Ichthyophthirius multifiliis isolate consisted of 6 to 10 trophonts collected by pipette as they dropped off infected goldfish placed in $3 \mathrm{I}$ aquaria against a black background. These were fixed and processed as for C. irritans isolates. DNA extracts were amplified by polymerase chain reaction (PCR), and sequenced by the dideoxy chain termination method as described previously (Adlard et al. 1993). The first internal transcribed spacer (ITS1) of the ribosomal RNA gene and the flanking 3' end of the $18 \mathrm{~S}$ region was amplified by PCR using oligonucleotide primers 1 (forward primer - 5' GTT CCC CTT GAA CGA GGA ATT C) and 2 (reverse primer - 5' CGC ATT TCG CTG CGT TCT TC). Primer 1 was located approximately 230 bp upstream of the 3 ' end of the 18S/TTS1 boundary while primer 2 was located in the $5.8 \mathrm{~S}$ region. Primers were designed for conserved regions from published sequieñces \{Hillis \& Dixon 1901).

Primer 2 and 2 other primers located within the amplified fragment were used to determine nucleotide sequences. The sequencing primers were primer 3 (forward primer - 5' GTC CCT GCC CTT TGT ACA CA) and primer 4 (reverse primer - 5' GAT CCT TCT

C.irr. CTTGAACGAGGAATCCTAGTAAGTGCAAGTCATCAGCTTGTACTGATTACGTCCCTGCCCTTTGTACACACCGC 75 I.mul. CTTGAACGAGGAATTCCTAGTAAGTGCAAGTCATCAGCTTGCGTTGATTATGTCCCTGCCGTTTGTACACACCGC T.pyr. CTTGAACGAGGAATTCTAGTAAGTGCAAGTCATCAGCTTGCGTTGATTATGTCCCTGCCGTTTGTACACACCGC T.aus. CTTGAACGAGGAATTCTAGTAAGTGCAAGTCATCAGCTTGCGTTGATTATGTCCCTGCCGTTTGTACACACCGC T.can. CTTGAACGAGGAATTCTAGTAAGTGCAAGTCATCAGCTTGCGTTGATTATGTCCCTGCCGTTTGTACACACCGC Co.in. CTTGAACAAGGAATTCTAGTAAGCATAAGTCATCAGCTTGTGCTGATTACGTCCCTGCCCTTTGTACACACCGC o.gra. CTTGAACGAGGAATTCCTAGTAAGCGCAAGTCATTACCTTGCGCTGATTAAGTCCCTGCCCTTTGTACACACCGC On.qu. CTTGAACGAGGAATTCCTAGTAAGCGCAAGTCATTAGCTTGCGCTGATTAAGTCCCTGCCCTTTGTACACACCGC

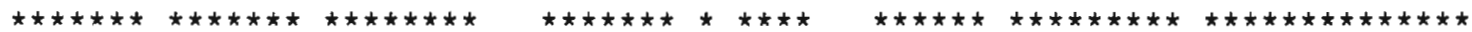

C.irr. CCGTCGCTCCTACCGATTTCGAGTATCCGGTGAACCTTCTGGACTGCGCTAACAXTAG---TTAGTGCGGGAAG 150

I.mu1. CCGTCGCTTGTAGTAA---CGAATGGTCTGGTGAACCTTCTGGACCGAGGTCG----CAAGGC---TTTGGGAAG

T.pYr. CCGTCGCTTGTAGTAA---CGAATGGTCTGGTGAACCTTCTGGACTGCGGTAG----CAATAC---TGCGGGAAA

T.aus. CCGTCGCTTGTAGTAA---CGAATGGTCTGGTGAACCTTCTGGACTGTGACAG----CAATGT---TACGGAAAA

T.can. CCGTCGCTTGTAGTAA---CGAATGGTCTGGTGAACCTTCTGGACTGCGGTAG----CAATAC---TGCGGAAAA

Co.in. CCGTCGCTCCTACCGATTTTGAGTGATCCGGTGAACCTTCTGGACTGTGGTCAGGCTTGACCTGATTGTGGGAAG

o. gra. CCGTCGCTCCTACCGATTTCGAGTGGTCCGGTGAACCTT'TTGGACTGCGCGAGGCCCCGAGCCTTGTGCGGAAAA On.qu. CCGTCGCTCCTACCGATTTCGAGTGATCCGGTGAACCATTTGGACTGCG-AGGTCCTCGTGACTT-TGTGGAAAA

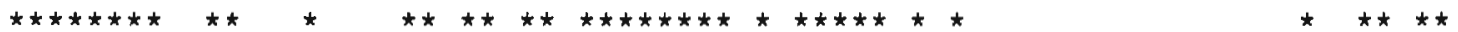

C. irr. TTAAGTAAACCACTTCACTTAGAGgAAgGAGAAGTCGTAACAAGgTTTCCGTAGgTGAACCTGCGGAAGGATCA I.mul. TTAAGTAAACCCTACCATTTGGAACAACAAGAAGTCGTAACAAGGTATCTGTAGGTGAACCTGCAGATGGATCA T.pyr。 ATAAGTAAACCCTACCATTTGGAACAACAAGAAGTCGTAACAAGGTATCTGTAGGTGAACCTGCAGATGGATCA T.aus. ATAAGTAAACCTACCATTTGGAACAACAAGAAGTCGTAACAAGGTATCTGTAGGTGAACCTGCAGATGGATCA T.can. ATAAGTAAACCCTACCATTTGGAACAACAAGAAGTCGTAACAAGGTATCTGTAGGTGAACCTGCAGATGGATCA Co.in. TTAAGTAAACCTTATCACTTAGAGGAAGGAGAAGTCGTAACAAGGTTTCCGTAGGTGAACCTGCAGAAGGATCA 0.gra. TCAAGTAAACCATATCACTTAGAGGAAGGAGAaGTCGTAACAAGgTTTCCGTAGgTGAACCTGCAGAAGgATCA on.qu. TCTAGTAAACCATATCACTTAGAGGAAGGAGAAGTCGTAACAAGGTTTCCGTAGGTGAACCTGCAGAAGGATCA

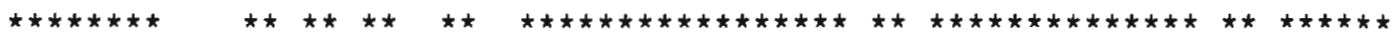

Fig. 1 Clustal $V$ alignment of nucleotide sequences beginning at the $5^{\prime}$ end of the amplified fragment. Base number 224 corresponds with the $3^{\prime}$ end of the $18 \mathrm{~S}$ region. Dashes (-) indicate alignment gaps, asterisks ( $\star$ ) indicate where bases are identical throughout the alignment. $\mathrm{X}=$ variable base. $C$. Irr. $=$ Cryptocaryon irritans, $I$. mul. $=$ Ichthyophthirius multifiliis, $T$. pyr. $=$ Tetrahymena pyriformis, $T$ aus. $=T$. australis, $T$ can $=T$ canadensis, Co. in $=$ Colpoda inflata, O. gra. = Oxytricha granulifera, On. qu. = Onychodromus quadricornutus 
GCA GGT TCA CCT AC), For all isolates, at least 1 replicate isolate was extracted, amplified and sequenced. Both the sense and non-sense strands were sequenced to further validate results

Sequences of Ichthyophthirius multifilis and Cryptocaryon irritans were compared to known sequences stored in GenBank using the Basic Local Alignment Search Tool (BLAST) routine (Altschul et al. 1990) available through the Australian National Genomic Information System (ANGIS). The 3 closest sequences to each were then used for comparison after alignment. The sequences obtained from GenBank were from the ciliates Colpoda inflata (from Greenwood et al. 1991), Oxytricha granulifera and Onychodromus quadricornutus (from Schlegel et al. 1991), and Tetrahymena pyriformis, $T$. canadensis and $T$. australis (from Sogin et al. 1986). Alignments were made by eye using the sequence editor ESEE (Cabot \& Bekenbach 1989) and verified using the alignment program Clustal V (Higgins et al. 1992). Alignment gaps were treated as missing bases.

Trees were constructed using maximum parsimony methods in PAUP ver. 3.0s (Swofford 1991). Bootstrap resampling of the data was used to indicate the degree of support for each branch of the tree.

\section{RESULTS}

Sequences were obtained for 224 bases from the 3' end of the $18 \mathrm{~S}$ region. In this region 66 bases were variable, of which 47 were informative. The sequence alignments used for analysis (Fig. 1) generated by Clustal $V$ were identical to those alignments adjusted by eye. Parsimony analyses using PAUP generated 2 most-parsimonious trees each 102 steps long (branch and bound search, consistency index 0.840 excluding uninformative characters) which differed only in the arrangement of the Tetrahymena spp. (Figs. $2 \& 3$ ). The hypotrichous ciliate Onychodromus quadricornutus was the designated outgroup in these analyses. Two major groupings of taxa were evident in both trees generated. One group consisted of Cryptocaryon irritans and Colpoda inflata, whilst the other contained Ichthyophthirius multifiliis and Tetrahymena pyriformis, T. canadensis and T. australis. Bootstrap resampling, which consisted of 1000 heuristic bootstrap replicate samples of the sequence data, indicated that support for these 2 separate groups was high (81\%), with $77 \%$ support for the C. irritans/Co. inflata group and $100 \%$ support for the $I$. multifiliis/Tetrahymena spp. group (Fig. 4). There was one variable base (position number 131 in Fig. 1) found between all 3 isolates of $C$. irritans sequenced, and no variation between the sequences of the 2 isolates of $I$. multifiliis.

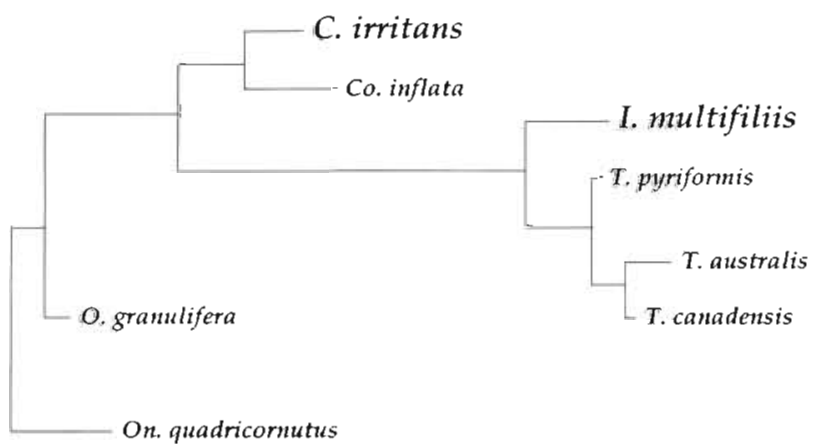

Fig. 2. The first of 2 most-parsimonious trees generated by the maximum parsimony analysis program PAUP. Onychodromus quadricornutus is the designated outgroup

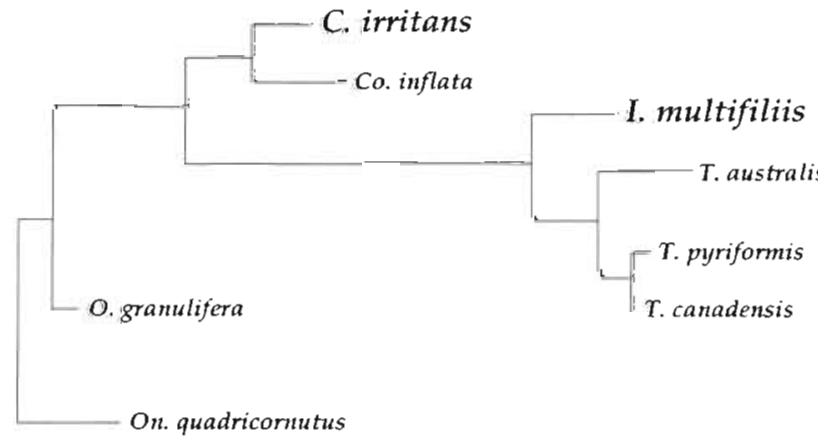

Fig. 3. The second of the most-parsimonious trees, differing from Fig. 2 only in the positioning of the Tetrahymena species $T$. pyriformis and $T$ australis

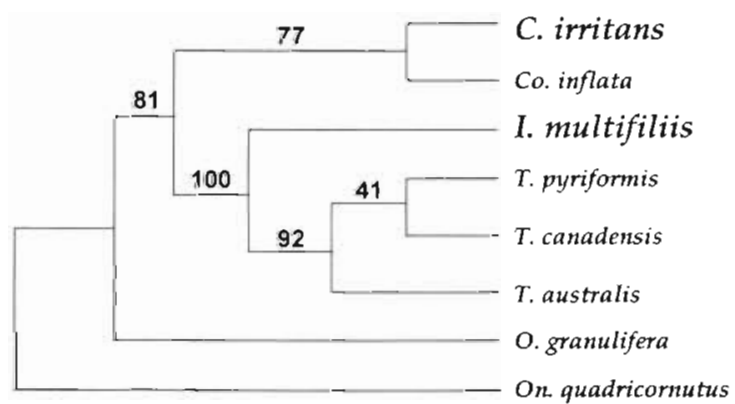

Fig. 4. Percentage support for each branch derived from 1000 heuristic bootstrap replicate samples of the sequence data

\section{DISCUSSION}

There was strong support for the grouping of Ichthyophthirius multifilis with the 3 species of Tetrahymena to the exclusion of Cryptocaryon irritans. This rRNA gene sequence data supports the present taxonomic placing by Corliss (1979) and Small \& Lynn (1985) of I. multifiliis (Suborder Ophyroglenina, Family Ichthyophthiriidae) within the Order Hymenostom- 
atida, Class Oligohymenophorea along with the Suborder Tetrahymenina (containing the 3 Tetrahymena species), but refutes the placing of $C$. irritans within the Family Ichthyophthiriidae. From this data, the Family Ichthyophthiriidae containing $C$. irritans is not seen to form a monophyletic group.

The placement of Cryptocaryon irritans in the Order Hymenostomatida has previously been questioned on morphological grounds (Cheung et al. 1981) due to the simplicity of its buccal apparatus which consists of a cytostome surrounded by a ring of cirri-like structures and lacks the distinct buccal cavity and oral membranelles of typical hymenostome ciliates. Its placement in the Suborder Ophyroglenina is also questionable as it does not possess an organelle of Lieberkühn ('watch-giass organelie') di any siaye of iis life historiy (Brown 1951), which contradicts the view of Lynn et al. (1991) who regarded the organelle of Lieberkühn as a synapomorphy of the Ophyroglenina.

Recent ultrastructural studies have revealed differences between the 2 species which also indicates that they are not as closely related as first thought. Colorni \& Diamant (1993) examined the development of trophonts, tomonts and theronts of Cryptocaryon irritans utilising TEM and found that the trophont possesses monokinetid somatic ciliature and an electrondense 'foamy' substance in the pellicular alveoli. The origin and function of the foamy substance was not clear, but this substance is not present in Ichthyophthirius multifiliis. They concluded that the development of $C$. irritans and $I$. multifiliis differed significantly. Matthews et al. (1993) found a similar electron-dense material in the pellicular alveoli of trophonts, tomites and theronts of $C$. irritans, and found that its mucocysts differed from those of $I$. multifiliis in size, shape and distribution. These mucocysts also appeared not to be directly involved in encystment as found for 1 . multifiliss (Ewing et al. 1983).

Our results, along with the morphological and ultrastructural evidence described above lend further support to the theory of Colorni \& Diamant (1993), who suggest that the superficial similarities between Ichthyophthirius multifilis and Cryptocaryon irritans are due to an adaptive convergence of life histories rather than phylogenetic proximity.

From the sequence data of the species available to us, Cryptocaryon irritans was consistently grouped in these analyses with Colpoda inflata. The genus Colpoda was recently placed in the new Class Colpodea (O. Colpodida) by Small \& Lynn (1985). Data obtained by analysis of 18 S rRNA (Lynn \& Sogin 1988) supported the Class status of the Colpodea and indicated that the colpodids may be a deeply split sister group of the Oligohymenophorea (which includes Tetrahymena and Ichthyophthirius multifiliis). The grouping of $C$. irritans with a colpodid in this study (although as a consequence of the close grouping of Ichthyophthirius and the Tetrahymena spp.) is nonetheless interesting given the similarities between the life histories of these taxa, with both $C$. irritans and many colpodids exhibiting encystment and reproduction by palintomy within the resting cyst. The simple buccal apparatus of $C$. irritans is also similar in appearance to the cytostomes found in some colpodids (for example Rostrophyra camerounensis; see Njine 1979). However, the fine structure of the somatic kinetid of colpodids is of a unique dikinetid construction (Lynn \& Small 1990) and presently little is known about the structure of the somatic kinetid of $C$. irritans except that it is of monokinetid construction (Colorni \& Diañiâît 1993).

Both the ultrastructural evidence and our sequence data suggest that the taxonomic position of Cryptocaryon irritans is distinct from that of Ichthyophthirius multifiliis. The association with the Colpodea suggested by the sequence data is interesting and has some support from morphological and life history viewpoints. However, no authoritative taxonomic placement of $C$. irritans can be performed until more information is available on the fine ultrastructure of its somatic kinetid. Important taxonomic information may also be revealed by silver staining techniques, as these methods have been underutilized in past studies of $C$. irritans. Also, the future availability of complete $18 \mathrm{~S}$ sequence data for $C$. irritans and an increased range of species of key ciliates (such as other colpodids and Ophryoglena) will provide for a clearer understanding of the taxonomic affinities of $C$. irritans.

Acknowledgements. The authors thank Dr Angelo Colorni for his prompt assistance in sending samples of fixed Cryptocaryon from Israel; also Dr Bob Lester and Dr Peter O'Donoghue for their comments on the manuscript and Dr Steve Barker for his assistance with the phylogenetic analysis. This work was supported in part by an Australian Postgraduate Research Award, and forms part of the doctoral thesis of B.K.D.

\section{LITERATURE CITED}

Adlard RD, Barker SC, Blair D, Cribb TH (1993) Comparison of the second internal transcribed spacer (ribosomal DNA) from populations and species of Fasciolidae (Digenea). Int J Parasitol 23:423-425

Altschul SF, Gish W, Miller W, Myers EW, Lipman DJ (1990) Basic local alignment search tool. J molec Biol 215: 403-410

Brown EM (1951) A new parasitic protozoan, the causal organism of a white spot disease in marine fish Cryptocaryon irritans gen \& sp.n. Agenda of Scientific Meetings of the Zoological Society, London, 1950, 11:1-2

Cabot EL, Bekenbach AT (1989) Simultaneous editing of multiple nucleic acid and protein sequences with ESEE. 
Comput Appl Biosci 5:233-234

Canella MF (1972) Ce qu'on ne connait pas sur un holotriche ectoparasite des poissons marins, découvert par le Dr Sikama et appelé Cryptocaryon irritans par Miss Brown. Ann Univ Ferrara Sez III Biol Anim 3:107-132

Cheung PF, Nigrelli RF, Ruggeri GD (1981) Scanning electron microscopy on Cryptocaryon urritans Brown 1951, a parasitic ciliate in marine fishes. J Aquaricult Kansas 2: $70-72$

Colorni A (1985) Aspects of the biology of Cryptocaryon irritans, and hyposalinity as a control measure in cultured gilt-head sea bream Sparus aurata. Dis aquat Org 1:19-22

Colorni A, Diamant A (1993) Ultrastructural features of Cryptocaryon irritans, a ciliate parasite of marine fish. Europ $J$ Protistol 29:425-434

Corliss JO (1975) Taxonomic characterization of the suprafamilial groups in a revision of recently proposed schemes of classification for the Phylum Ciliophora. Trans A.m Microsc Soc 94:224-267

Corliss JO (1979) The ciliated Protozoa, 2nd edn. Pergamon Press, Oxford

de Puytorac P (1994) Infusoires ciliés, Fascicule 2 Systématique. In: Grassé PP (ed) Traité de zoologie, anatomie, systématique, biologie, Tome II. Masson, Paris, p 813-822

Ewing MS, Kocan KM, Ewing SA (1983) Ichthyophthirius multifiliis: morphology of the cyst wall. Trans Am Microsc Soc 102:122-128

Greenwood SJ, Schlegel M, Sogin ML, Lynn DH (1991) Phylogenetic relationships of Blepharisma americanum and Colpoda inflata within the Phylum Ciliophora inferred from complete small subunit rRNA gene sequences. J Protozool 38:1-6

Higgins DG, Bleasby AJ, Fuchs R (1992) CLUSTAL V: improved software for multiple sequence alignment. Comput Appl Biosci 8:189-191

Hillis DM, Dixon MT (1991) Ribosomal DNA: molecular evolution and phylogenetic inference. Q Rev Biol 66:411-453

Huff JA, Burns CD (1981) Hypersaline and chemical control of Cryptocaryon irritans in red snapper, Lutjanus campechanus monoculture. Aquaculture 22:181-184

Kaige N, Miyazaki T (1985) A histopathological study of white spot disease in Japanese flounder. Fish Pathol 20:61-64 (in Japanese, English summary)

Lom J, Dykova I (1992) Protozoan parasites of fishes. Elsevier, Amsterdam

Lynn DH, Small EB (1990) Phylum Ciliophora. In: Margulis L, Corliss JO, Melkonian M. Chapman DJ (eds) Handbook of Protoctista. Jones and Bartlett, Boston, p 498-523

Lynn DH, Sogin ML (1988) Assessment of phylogenetic relationships among ciliated protists using partial ribosomal RNA sequences derived from reverse transcripts. BioSystems 21:249-254

Lynn DH, Frombach S, Ewing MS, Kocan KM (1991) The organelle of Lieberkühn as a synapomorphy for the

Responsible Subject Editor: W. Körting, Hannover, Germany
Ophyroglenina (Ciliophora: Hymenostomatida). Trans Am Microsc Soc 110:1-11

Matthews BF, Matthews RA, Burgess PJ (1993) Cryptocaryon irritans Brown, 1951 (Ichthyophthiriidae): the ultrastructure of the somatic cortex throughout the life cycle. J Fish Dis 16:339-349

Nigrelli RF, Ruggeri GD (1966) Enzootics in the New York Aquarium caused by Cryptocaryon irritans Brown, 1951 (= Ichthyophthirius marinus Sikama, 1961), a histophagous ciliate in the skin, eyes and gills of marine fishes. Zoologica 51:97-102

Nigrelli RF, Pokorny KS, Ruggeri GD (1976) Notes on Ichthyophthirius multifiliis, a ciliate parasite on freshwater fishes, with some remarks on possible physiological races and species. Trans Am Microsc Soc 95:607-613

Njine T (1979) Compléments a l'étude des ciliés libres du Cameroun. Protistologica 15:343-354

Rasheed VM (1989) Diseases of cultured brown-spotted grouper Epinephelus tauvina and silvery black porgy Acanthopagrus cuvieri in Kuwait. J Aquat Anim Health 1: $102-107$

Sambrook J, Fritsch EF, Maniatis T (1989) Molecular cloning; a laboratory manual, 2nd edn. Cold Spring Harbour Laboratory Press, Cold Spring Harbour, New York

Schlegel M, Elwood HJ, Sogin ML (1991) Molecular evolution in Hypotrichous ciliates: sequence of the small subunit ribosomal RNA genes from Onychodromus quadricornutus and Oxytricha granulifera (Oxytrichidae, Hypotrichida, Ciliophora). J molec Evol 32:64-69

Sikama Y (1937) Preliminary report on the white spot disease in marine fish. Suisan Gakukai 7:149-160 (in Japanese)

Sikama Y (1961) On a new species of Ichthyophthirius found in marine fishes. Sci Rep Yokosuka City Mus 6:66-70

Small EB, Lynn DH (1985) Phylum Ciliophora. In: Lee JJ, Hutner SH, Bovee EC (eds) An illustrated guide to the protozoa. Society of Protozoology. Lawrence, p 393-575

Sogin ML, Elwood HJ (1986) Primary structure of the Paramecium tetraurelia small subunit rRNA coding region: phylogenetic relationships within the Ciliophora. J molec Evol 23:53-60

Sogin ML, Ingold A, Karlok M, Nielsen H, Engberg J (1986) Phylogenetic evidence for the acquisition of ribosomal RNA introns subsequent to the divergence of some of the major Tetrahymena groups. EMBO J 5:3625-3630

Swofford DL (1991) PAUP: phylogenetic analysis using parsimony (Version 3.0s). Computer program distributed by Illinois Natural History Survey, Champaign, IL

Wilkie DW, Gordin H (1969) Outbreak of cryptocaryoniasis in marine aquaria at Scripps Institution of Oceanography. Calif Fish Game 55:227-236

Wurtsbaugh WA, Tapia RA (1988) Mass mortality of fishes in Lake Titicaca (Peru-Bolivia) associated with the protozoan parasite Ichthyophthirius multifiliis. Trans Am Fish Soc 117:213-217

Manuscript first received: July 12, 1994

Revised version accepted: December 23, 1994 